\title{
2020 Reviewer Thank You
}

CARTILAGE

202I, Vol. I2(2) 263-265

(C) The Author(s) 2021

Article reuse guidelines:

sagepub.com/journals-permission DOI: 10.1 I77/I947603521993248

journals.sagepub.com/home/CAR

QSAGE
Abrar, Daniel

Adam, Emma

Ainsworth, Madison

Akeda, Koji

Al-Shammari, Ahmed

Alibegović, Armin

Almqvist, Fredrik

Altman, Roy

Amendola, Annunziato

Angele, Peter

Årøen, Asbjørn

Aurich, Matthias

Balain, Birender

Band, Philip

Bank, Ruud

Barreto-Henriksson, Helena

Battistelli, Michela

Bay-Jensen, Anne-Christine

Becher, Christoph

Behrens, Peter

Beier, Frank

Belluzzi, Elisa

Biant, Leela

Blums, Kristaps

Blunk, Torsten

Bohndorf, Klaus

Bonassar, Lawrence

Bonner, Kevin

Bos, Koen

Boutet, Marie-Astrid

Brinchmann, Jan E.

Brophy, Robert

Buford, Don

Bugbee, William

Caam, Arjan

Carey, James

Caron, Marjolein

Cavallo, Carola

Chandrasekharan, U

Chapman, Cary

Chaudhari, Akshay Sanjay

Che, Adeelah

Chevalier, Xavier

Christensen, Bjørn

Chubinskaya, Susan

Cilleropastor, Berta

Cohen, Moisés
Cole, Brian

Collard, Jean-Francois

Conley, Caitlin

Convill, James

Cook, James

Cornish, Stephen

Cottrell, Jessica

Coury, Josephine

Coveney, Clarissa

Creemers, Laura

Cristiani, Riccardo

Custers, Roel

Dahmen, Jari

Dawson, Matt

De Boer, Nans

Deberardino, Thomas

Decaro, Francesca

Di Matteo, Berardo

Díaz Payno, Pedro

Diekman, Brian

Dodge, George

Donnelly, Patrick

Doria, Carlo

Dorotka, Ronald

Dwivedi, Garima

Dyment, Nathaniel

Eckstein, Felix

Ekman, Stina

Engebretsen, Lars

Enomoto-Iwamoto, Motomi

Ettinger, Sarah

Evans, Adam

Fahy, Niamh

Farquharson, Colin

Farr, Jack

Felländer-Tsai, Li

Ferkel, Richard

Ferrao Blanco, Mauricio

Filardo, Giuseppe

Francisco, Vera

Freitag, Julien

Frondoza, Carmelita

Fuchs, Christiane

Fuggle, Nicholas

Fulkerson, John

Gatenholm, Birgitta

Gersoff, Wayne 
Geurts, Jeroen

Gille, Justus

Gobbi, Alberto

Goldring, Mary

Goldstein, Todd

Gomoll, Andreas

Grad, Sibylle

Grande, Daniel

Grant, John

Grässel, Susanne

Griffin, Timothy

Grigolo, Brunella

Grogan, Shawn

Gudeman, Andrew

Guermazi, Ali

Guilak, Farshid

Gursoy, Safa

Hall, Andrew

Hammoud, Sommer

Hamrin-Senorski, Eric

Hardingham, Tim

Haudenschild, Dominik

Hauzeur, Jean-Philippe

Henrotin, Yves

Hevesi, Mario

Hollander, Anthony

Houard, Xavier

Hughes, Clare

Hui, James

Hurley, Eoghan

Hurtig, Mark

Ichikawa, Masakazu

Ike, Robert

Irwin, Rebecca

Ivanovska, Ana

Jandačka, Daniel

Jay, Gregory

Jenei-Lanzl, Zsuzsa

Jeon, Hyunjae

Jeyakumar, Vivek

Johnstone, Brian

Joseph, Gabby

June, Ronald

Juras, Vladimir

Kafienah, Wael

Kandel, Rita

Karlsson, Jon

Kennedy, John

Kisiday, John

Koff, Matthew

Kogan, Feliks

Kölby, Lars

Kon, Elizaveta

Korpershoek, Jasmijn
Kraan, Pm

Krych, Aaron

Kuiper, Jan Herman

Kumar, Pavan

Lafeber, Floris

Lammi, Mikko

Laprade, Robert

Lattermann, Christian

Laverty, Sheila

Lim, Chee Kim

Lim, Khoon

Lind, Martin

Lindahl, Anders

Link, Thomas

Liu, Gang

Liu, X

Loebel, Claudia

Lording, Timothy

Lotz, Martin

Malaise, Olivier

Malemud, Charlie

Malfait, Anne-Marie

Mandelbaum, Bert

Mansfield, J C

Massari, Leo

Mastbergen, Simon

Mccarrel, Taralyn

Mccoy, Annette M.

Mckenna, Frank

Melugin, Heath

Michelacci, Yara

Miller, April

Minas, Tom

Mithoefer, Kai

Mlynarik, Vladimir

Mobasheri, Ali

Mumme, Marcus

Murphy, Mary

Nakamura, Norimasa

Nehrer, Stefan

Nelson, Brad

Nelson, Fred

Nicholls, Mathew

Nykänen, Olli

Ortved, Kyla

Ossendorff, Robert

Pachowsky, Milena

Paget, Liam

Parikh, Shital

Patel, Jay

Paton, Robin

Pattappa, Girish

Pest, Mike

Philippon, Marc 
Philips, M

Pilmane, Mara

Pisanu, Francesco

Piuzzi, Nicolas

Plaas, Anna

Plancher, Kd

Pleshko, Nancy

Potter, Hollis

Pritzker, Ken

Ramos, Yolande

Randolph, Mark

Randsborg, Per-Henrik

Rannou, François

Ribeiro, Joao

Roberts, Sally

Rodeo, Scott

Roemer, Frank

Roseti, Livia

Saarakkala, Simo

Sah, Robert

Salzmann, Gian

Sanchez, Mikel

Saris, Daniel

Saxne, Tore

Schmaranzer, Florian

Schneider, Cornelia

Schreiner, Markus

Seitz, Andreas

Serra, C. Ivan

Sgaglione, Nicholas

Shea, Kevin

Sherman, Seth

Simonsson, Stina

Singh, Purva

Snow, Martyn

Sommer, Nicole

Spalding, Tim

Squillace, Dominic
Stevens, Hazel

Stoddart, Martin

Strauss, Eric

Strickland, Sabrina

$\mathrm{Su}, \mathrm{Nan}$

Szomolanyi, Pavol

Tanska, Petri

Tasso, Roberta

Tew, Simon

Toh, Wei Seong

Trattnig, Siegfried

Utomo, Lizette

Vaira, Luigi

Van Buul, Gerben

Van Den Akker, Guus

Van Heerwaarden, Ronald

Van Wijnen, Andre

Verdonk, René

Vernerey, Franck

Vidal, Armando

Von Rechenberg, Brigitte

Vonk, Lucienne

Walsh, Shannon

Welsch, Goetz

Welting, Tim

Wikstrom, Erik

Williams, Andy

Wing Shan Sit, Regina

Wollstein, Ronit

Woodell, Jennifer

Xiao, Jianqui

$\mathrm{Xu}$, Shiwen

Yadav, S

Yanke, Adam

Yu, Fangfang

Zaffagnini, Stefano

Zippelius, Timo 